\title{
Área de vida de Cistothorus platensis (Aves: Troglodytidae) no Cerrado brasileiro
}

\author{
Aline Fujikawa' \& Dárius P. Tubelis² [D
}

\begin{abstract}
1. Universidade de São Paulo, Instituto de Biociências, Departamento de Ecologia, Rua do Matão, 321, Cidade Universitária, 05508-090 São Paulo, SP, Brasil. (alinefujikawa84@gmail.com)

2. Universidade Federal Rural do Semi-Árido, Departamento de Biociências, Av. Francisco Mota, 576, Bairro Costa e Silva, 59625-900 Mossoró, RN, Brasil. (darius.tubelis@gmail.com)
\end{abstract}

Recebido 3 abril 2019

Aceito 11 novembro 2019

Publicado 22 maio 2020

DOI 10.1590/1678-4766e2020006

\begin{abstract}
Home range of Cistothorus platensis (Aves: Troglodytidae) in the Brazilian Cerrado. The Sedge Wren Cistothorus platensis (Latham, 1790) is a widely distributed bird species, occurring in natural grasslands through the American continent. Most knowledge on its biology has been obtained in temperate regions, and no information on its home range in tropical grasslands is available. The goal of this study was to examine the home ranges of Sedge Wrens at the Parque Nacional da Chapada dos Veadeiros, central Cerrado, Brazil. Fourteen banded males were studied in a patch of shrubby grassland (campo sujo) between February and December 2008. The mean size of home ranges of five males monitored during the whole period was $6.10 \pm 2.09$ ha (Minimum Convex Polygon) and 4.57 \pm 1.92 ha (Kernel 95\%). Home ranges were larger in the dry season (non-breeding period) than in the rainy season (that encompassed both the breeding and non-breeding periods). The overlap between home ranges of neighboring males was small. Sedge Wrens did not establish home ranges on recently burned grasslands. Five banded males were recorded during the entire study period, suggesting the existence of resident individuals. Nine of the 14 males did not persist in the same site throughout the year. There was no evidence of polygyny, as only lone birds or pairs were detected. Our study indicates that Sedge Wrens inhabiting grasslands of the Cerrado are monogamous and have larger home ranges than in temperate regions.
\end{abstract}

KEYWORDS. Grasslands, Neotropical Region, savanna, Wren.

RESUMO. A corruíra-do-campo Cistothorus platensis (Latham, 1790) apresenta ampla distribuição geográfica, ocorrendo ao longo de todo o continente americano. Informações sobre sua biologia têm sido obtidas principalmente em regiões temperadas, e o conhecimento sobre suas áreas de vida em campos tropicais é inexistente. O objetivo deste trabalho foi estudar as áreas de vida de C. platensis no Parque Nacional da Chapada dos Veadeiros, Cerrado do Brasil central. Quatorze machos foram anilhados em um campo sujo e cinco deles puderam ser acompanhados continuamente entre fevereiro e dezembro de 2008. O tamanho médio das áreas de vida $(\mathrm{n}=5)$ foi de $6,10 \pm 2,09$ ha (Mínimo Polígono Convexo) e 4,57 $\pm 1,92$ ha $($ Kernel $95 \%)$ e variaram significativamente entre as estações estudadas. As áreas de vida foram maiores na estação seca (período não-reprodutivo) do que na estação chuvosa (que abrangeu os períodos reprodutivo e não-reprodutivo). Houve baixa sobreposição entre áreas de machos vizinhos. As aves estudadas não estabeleceram áreas de vida em trechos de campo recentemente queimados. Alguns indivíduos foram registrados durante todo o período do estudo, sendo assim considerados residentes. Entretanto, nove dos 14 machos não persistiram no local. Não houve evidência de poliginia, uma vez que somente casais ou aves solitárias foram registradas. Este estudo indica que C. platensis habitando campos naturais no Cerrado são monogâmicos e têm áreas de vida maiores do que em regiões temperadas.

PALAVRAS-CHAVE. Campos, corruíra, Região Neotropical, savana.

A área de vida pode ser definida como a área total na qual um indivíduo vive, buscando alimento, parceiros sexuais e abrigo (BuRT, 1943; Brown \& Orians, 1970). A análise da área de vida amplia o conhecimento sobre a biologia das espécies e disponibiliza informações importantes para estratégias de conservação e manejo (PYKE et al., 1977). Por exemplo, o conhecimento da área de vida de uma espécie é importante para a tomada de decisão relativa ao tamanho de reservas, garantindo a conservação de porções de hábitat necessários para a reprodução e a alimentação dos indivíduos (Preston et al., 1998).

Adicionalmente, o tamanho da área de vida é um importante atributo ecológico, pois pode determinar a densidade populacional, afetar os sistemas de acasalamento e influenciar a distribuição e a abundância local de invasores e presas (CARPENTER, 1987). Estudos referentes a área de vida de aves da Região Neotropical estão disponíveis para diversas espécies (e.g., AMARAL \& MACEDO, 2003; LOPES \& Marini, 2006; Freitas \& Rodrigues, 2012; Duca \& MARINI, 2014; SouZA et al., 2018). Entretanto, espécies da família Troglodytidae carecem de estudos.

A corruíra-do-campo Cistothorus platensis (Latham, 1790) é um trogloditídeo amplamente distribuído nas Américas, habitando regiões como os Andes, a Patagônia, os campos do Rio da Prata e o Cerrado (Del Hoyo et al., 2005; Llambías et al., 2018). Esta espécie de ave insetívora 
e campestre, que não se adapta a áreas modificadas (SICK, 1997; VicKerY et al., 1999; Tubelis \& CAVALCANTI, 2001), teve asptectos de sua biologia investigados principalmente na América do Norte (BuRNs, 1982; KroOdSMA et al., 1999a; Del Hoyo et al., 2005; KIRSCH, 2007) e Argentina (LlambÍAS et al., 2018).

Importantes diferenças comportamentais no sistema de acasalamento e nos padrões de migração e vocalização foram verificadas entre as populações que ocorrem nas regiões temperada e tropical (KroodSMA et al., 1999b; DeL HoYo et al., 2005). No entanto, não existem informações sobre diferenças no tamanho das áreas de vida. Isto porque as áreas de vida de $C$. platensis foram estudadas somente na América do Norte (BuRNs, 1982; KIRSCH et al., 2007).

Diversos estudos constataram variações no tamanho das áreas de vida de aves entre as estações reprodutiva e nãoreprodutiva nas regiões temperada (e.g., Preston et al., 1998; VANDERWerf, 1998) e tropical (e.g., Amaral \& Macedo, 2003). Estes e outros trabalhos apontam como principais variáveis causadoras de tal variação a disponibilidade de recursos, o tamanho do grupo e a necessidade de cuidado do ninho e filhotes. Entretanto, não se sabe se tais variações se aplicam a $C$. platensis, visto que todos os estudos de áreas de vida dessa ave foram conduzidos durante o período reprodutivo (BURNs, 1982; KIRSCH et al., 2007).

O objetivo deste estudo foi examinar áreas de vida de C. platensis em um trecho de campo sujo no Parque Nacional da Chapada dos Veadeiros, estado de Goiás, Brasil central. Mais especificamente, foram respondidas as seguintes questões: (1) qual o tamanho da área de vida dessa espécie?; (2) há sobreposição nas áreas de vidas de indivíduos vizinhos?; (3) há diferenças no tamanho da área de vida em relação aos períodos reprodutivo e não-reprodutivo da ave e às estações seca e chuvosa do Cerrado?

\section{MATERIAL E MÉTODOS}

O Cerrado. Esta região savânica domina a região central do Brasil e estende-se através de penínsulas e manchas disjuntas às províncias vegetacionais vizinhas - Amazônia, Mata Atlântica e Caatinga (EITEN, 1972; OliveIRA-FilHo \& RATTER, 2002). As paisagens são geralmente dominadas por cerrado sensu lato - um gradiente de vegetação savânica que inclui campo limpo, campo sujo, campo cerrado, cerrado sensu stricto e cerradão. Este gradiente domina os interflúvios e é interrompido nos vales por vegetação associada a cursos d'água, tais como matas de galeria, veredas e brejos. Fitofisionomias menos dominantes são campos rupestres, matas secas, campos úmidos e veredas (EITEN, 1972; Castro \& Kauffman, 1998; Oliveira-Filho \& RATTER, 2002). O clima do Cerrado do Brasil central é marcadamente sazonal, apresentando duas estações bem definidas: uma seca, com início no mês de maio e término no mês de setembro, e outra chuvosa, que vai de outubro a abril (AssAD, 1994). Esta sazonalidade é evidente nas diferenças exibidas pela vegetação e pela abundância de insetos, frutos e flores (OliveIRA, 1998; MACEDO, 2002).
Parque Nacional da Chapada dos Veadeiros. As atividades de campo foram realizadas no Parque Nacional da Chapada dos Veadeiros, localizado no nordeste do estado de Goiás (Fig. 1). Este parque tem atualmente uma área de 65.512 ha $\mathrm{e}$ as altitudes variam entre $620 \mathrm{~m}$ e $1.650 \mathrm{~m}$ (ICMBio, 2019). A região apresenta um clima com precipitação média anual entre $1.500 \mathrm{~mm}$ e $1.750 \mathrm{~mm}$, concentrada entre os meses de outubro e abril (ASSAD, 1994; SiLVA et al., 2001). A temperatura média anual varia em torno de $24^{\circ}$ a $26^{\circ} \mathrm{C}$. O parque apresenta relevo de chapada, com solos rasos e predominância de vegetação típica associada, como campos limpos, campos sujos, campos rupestres e cerrado sensu stricto (Assad, 1994; Felfili et al., 2007).

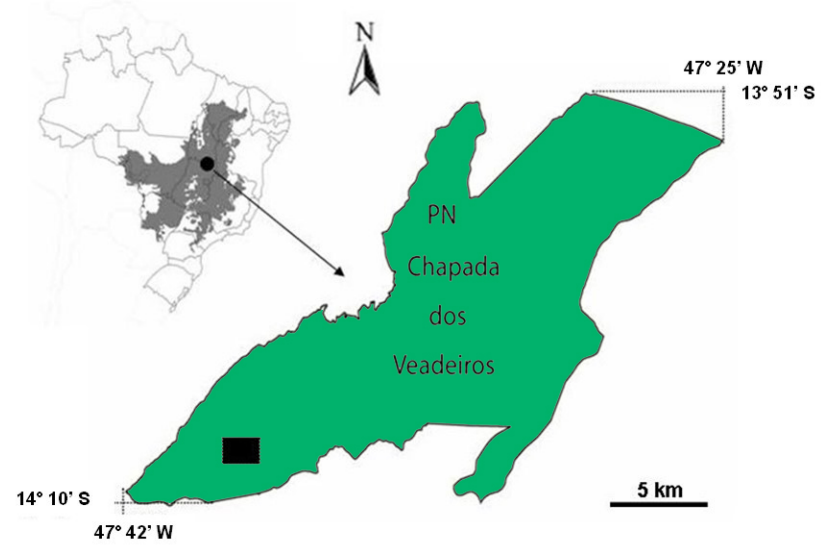

Fig. 1. Área de estudo (retângulo preto) onde áreas de vida de Cistothorus platensis (Latham, 1790) foram estudadas no Parque Nacional da Chapada dos Veadeiros, no Cerrado, Goiás (região mais escura sobre o Brasil), em 2008.

O campo sujo é uma fisionomia predominantemente herbáceo-arbustiva, com densa cobertura herbácea, numerosos arbustos e subarbustos esparsos (OLIVEIRA-FILHO \& RATTER, 2002). As famílias encontradas com mais frequência são Poaceae e Cyperaceae (RibeIro \& WALTER, 1998; FilgueIRAS, 2002). Muitas vezes, o estrato arbustivo é constituído por indivíduos menos desenvolvidos das espécies arbóreas de cerrado sensu stricto (RIBEIRo \& WALTER, 1998). Campos sujos ocorrem em solos rasos ou solos profundos de baixa fertilidade e areias quartzosas (RIBEIRO \& WALTER, 1998; Oliveira-Filho \& Ratter, 2002).

Captura e observação das aves. Indivíduos de $C$. platensis foram capturados na primeira quinzena de fevereiro de 2008 em um trecho $(800 \times 800 \mathrm{~m})$ de campo sujo de aproximadamente 1.000 ha localizado na região sul do parque (Fig. 1). As atividades de campo ocorreram entre os meses de fevereiro e dezembro de 2008. Foram realizadas observações em três períodos de amostragem: 1) "período não-reprodutivo - estação chuvosa" - 11 de fevereiro a 8 de abril. Corresponde à estação não-reprodutiva de $C$. platensis e ao período mais chuvoso na região; 2) "período não-reprodutivo - estação seca" - 21 de agosto a 30 de setembro. Corresponde à estação não-reprodutiva de $C$. platensis e ao período de seca na região; 3) "período reprodutivo" - 11 de outubro a 2 de 
dezembro. Corresponde à estação reprodutiva de C. platensis e ao período chuvoso na região.

Cada um desses períodos de amostragem foi composto por cerca de 20-25 dias de observações contínuas, seguidas por um intervalo de aproximadamente 15 dias sem observações e mais 10-15 dias de observações contínuas. Esta divisão adotada para os períodos reprodutivo e não-reprodutivo segue MARINI et al. (2012), que reportaram o encontro de um ninho com ovos em meados de novembro e outro com filhotes bastante desenvolvidos no fim de outubro em campos naturais do Distrito Federal.

Catorze machos de C.platensis foram capturados com uma rede de neblina. As aves capturadas receberam duas anilhas coloridas, uma em cada tarso, e foram rapidamente soltas ao lado da rede. As observações ocorreram em dois horários: manhã ( $06: 30 \mathrm{~h}$ às 09:00 h) e tarde (16:00 h às 18:00 h). Durante cada manhã ou tarde, machos anilhados foram procurados e, quando encontrados, tiveram sua localização registrada com GPS (erro nominal de $8 \mathrm{~m}$ ). Uma nova localização foi registrada a cada três minutos, seguindo orientação em De Solla et al. (1999), que sugerem que o número de observações seja maximizado utilizando-se intervalos constantes entre os pontos, aumentando assim a acurácia e a precisão das estimativas das áreas de vida.

Para evitar possíveis modificações de comportamento devido à presença do observador, os animais anilhados foram observados a distâncias superiores a $15 \mathrm{~m}$. O observador procurou ficar parado, observando a ave até perdê-la de vista, sem segui-la. Em seguida, outra ave anilhada foi procurada nas proximidades e o procedimento de observação repetido.

O esforço amostral total foi de 340 horas ao longo de 60 dias de observações realizadas entre fevereiro e dezembro de 2008. Apenas cinco dos 14 indivíduos anilhados foram observados durante todo o período de estudo. Os demais indivíduos não foram registrados por algumas semanas.

Cálculo das áreas de vida. Dois diferentes estimadores de área de vida foram utilizados: Mínimo Polígono Convexo (MPC) e Kernel. O primeiro consiste basicamente na união dos pontos mais extremos da distribuição de localizações, de forma a fechar o menor polígono possível sem admitir concavidades (WorTON, 1987; POWELl, 2000). O MPC é o mais antigo estimador de área de vida (ODUM \& KUENZLER, 1955). Este método foi utilizado devido à facilidade de aplicação e extenso uso, tornando mais fácil a comparação entre estudos (HARRIS et al., 1990). Por outro lado, usandose o estimador de Kernel são considerados os padrões de intensidade de uso do espaço através de um modelo baseado, principalmente, em funções de probabilidade de densidade (Worton, 1987; Powell, 2000). O Kernel é considerado um dos mais acurados estimadores para avaliar a distribuição das localizações dos indivíduos (Powell, 2000; JACOB \& Rudran, 2003; LAVER \& Kelly, 2008).

Seguindo-se as recomendações de SEAman et al. (1999) e LAVER \& KELLY (2008), foram calculados os Kernels fixos $95 \%$ e $50 \%$, referentes respectivamente, à área de vida e à área nuclear (trechos mais intensamente utilizados pelos indivíduos), com o fator de suavização $(h)$ calculado pelo método de validação cruzada de quadrados mínimos (Least squares cross-validation). A extensão Animal Movement (Hooge \& Eichenlaub, 2000) no Arcview 3.2 (ESRI, 1999) foi utilizada para delimitar e calcular as áreas de vida.

Somente as áreas de vida dos indivíduos cujas curvas das áreas de vida sazonais (de cada um dos três períodos de amostragem) e anuais (referente aos três períodos somados) atingiram a estabilização foram apresentadas. A estabilização foi verificada ao examinar a relação entre o tamanho da área e o número de localizações para um determinado período. Áreas foram consideradas estabilizadas quando localizações adicionais resultaram em um aumento mínimo na área, como em HARRIs et al. (1990). As curvas foram geradas através do Boostrap MPC 100\%, utilizando-se a extensão Animal Movement (Hooge \& Eichenlaub, 2000) no Arcview 3.2 (ESRI, 1999).

Análises estatísticas. As diferenças no tamanho das áreas de vida entre os três períodos de estudo foram verificadas com o teste do sinal aplicado a cada dupla de períodos. Todas as análises foram feitas de acordo com ZAR (2009) considerando um nível de significância de 5\%. O pacote estatístico RGui versão 2.8.5, foi utilizado para realizar as análises.

\section{RESULTADOS}

Áreas de vida. A curva de estabilização indicou que o número de pontos de localização obtidos foi suficiente para o cálculo de suas áreas de vida anuais (Fig. 2).

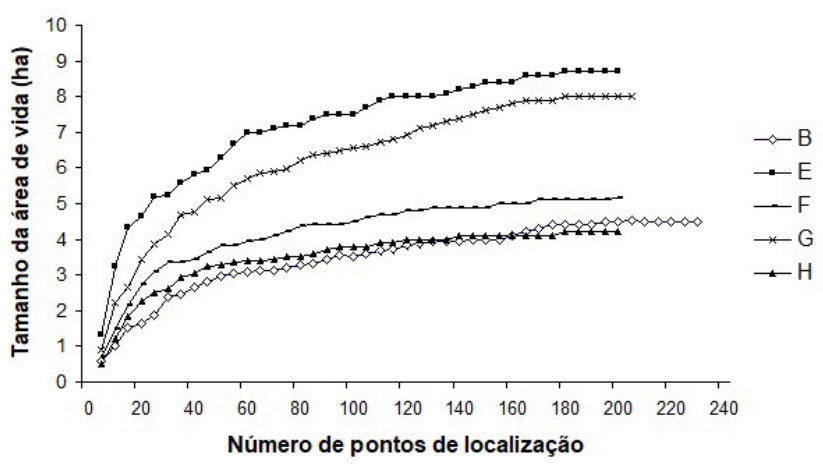

Fig. 2. Curva de estabilização do tamanho das áreas de vida de cinco indivíduos de Cistothorus platensis (Latham, 1790) em campo sujo no Parque Nacional da Chapada dos Veadeiros, Goiás, Brasil central, entre fevereiro e dezembro de 2008. As áreas de vida foram calculadas pelo método do Mínimo Polígono Convexo.

Machos de C. platensis $(n=5)$ tiveram áreas de vida anuais variando entre 4,15 ha e 8,67 ha, com média de $6,10 \pm$ 2,09 ha (MPC). As áreas de vida anuais calculadas pelo método de Kernel 95\% variaram entre 2,52 ha e 6,99 ha, com média de 4,57 $\pm 1,92$ ha. As áreas nucleares (Kernel $50 \%$ ) oscilaram entre 0,20 e 1,05 ha, com média de $0,48 \pm$ 0,34 ha (Tab. I).

No período não-reprodutivo - estação chuvosa, a média das áreas de vida $(\mathrm{n}=8)$ de C. platensis foi de $1,49 \pm$ 0,37 ha (MPC), 1,63 $\pm 0,33$ ha (Kernel $95 \%$ ) e 0,23 $\pm 0,07$ ha 
(Kernel 50\%). No período não-reprodutivo - estação seca, a média das áreas de vida $(n=10)$ foi de 3,47 $\pm 1,53$ ha (MPC), $3,92 \pm 1,74$ ha (Kernel 95\%) e 0,60 $\pm 0,41$ ha (Kernel 50\%); no período reprodutivo, a mesma média $(n=7)$ foi de $2,18 \pm$ 1,24 ha (MPC), 2,68 $\pm 1,30$ ha (Kernel 95\%) e 0,42 $\pm 0,16$ ha (Kernel 50\%) (Tab. II).

Considerando todos os indivíduos estudados e os três métodos utilizados (Tab. II), houve diferença significativa no tamanho das áreas de vida no período não-reprodutivo - estação seca e no período reprodutivo (MPC: $P=0,04$; Kernel 95\%: $P=0,04$; Kernel 50\%: $P=0,04)$. O tamanho das áreas de vida foi maior no período não-reprodutivo - estação seca seca do que na estação reprodutiva. Houve diferença significativa no tamanho das áreas de vida nos períodos não-reprodutivo das estações chuvosa e seca somente para o estimador Kernel 95\% (MPC: $P=0,22$; Kernel 95\%: $P=$ 0,03; Kernel 50\%: $P=0,06)$. Para este estimador, o tamanho das áreas de vida foi maior no período não-reprodutivo estação seca do que no período não-reprodutivo - estação chuvosa. Não houve diferença significativa entre os tamanhos das áreas de vida no período não-reprodutivo - estação chuvosa e no período reprodutivo (MPC: $P=1,00$; Kernel 95\%: $P=0,38$; Kernel 50\%: $P=0,38)$.

Houve baixa sobreposição entre as áreas de vida dos machos de C. platensis (Fig. 3). Considerando-se cada um dos períodos estudados (Tab. III), foi observada baixa sobreposição entre as áreas de vida no período nãoreprodutivo - estação chuvosa. As áreas de dois machos se sobrepuseram. Esta sobreposição representou 1,2\% (MPC) e 2,1\% (Kernel 95\%) da área de vida do macho D, e 1,2\% (MPC) e 1,6\% (Kernel 95\%) da área de vida do macho E.

No período não-reprodutivo - estação seca as áreas de vida de quase todos (MPC) e todos (Kernel 95\%) os machos se sobrepuseram à área de, pelo menos, um co-específico. No período reprodutivo, houve menor sobreposição (quatro de 10 indivíduos estudados). Não foi observada sobreposição entre as áreas nucleares no período não-reprodutivo - estação chuvosa e no período reprodutivo. No período reprodutivo estação seca, houve baixíssima sobreposição entre as áreas nucleares dos machos A $(1,8 \%)$ e $\mathrm{E}(3,5 \%)$, e entre os machos B $(4,1 \%)$ e $\mathrm{J}(6,6 \%)$.

Tab. I. Tamanho (ha) das áreas de vida anuais de cinco indivíduos de Cistothorus platensis (Latham, 1790) em campo sujo no Parque Nacional da Chapada dos Veadeiros, Goiás, Brasil central. As áreas de vida foram calculadas utilizando-se três diferentes estimadores: Mínimo Polígono Convexo (MPC), Kernel 95\% e Kernel 50\%.

\begin{tabular}{ccccc}
\hline \multirow{2}{*}{$\begin{array}{c}\text { Indivíduos } \\
\text { estudados }\end{array}$} & $\begin{array}{c}\text { Número de } \\
\text { pontos obtidos }\end{array}$ & MPC & Tamanho das áreas de vida \\
\cline { 3 - 5 } B & 229 & 4,51 & Kernel 95\% & Kernel 50\% \\
\hline E & 205 & 8,67 & 6,52 & 0,24 \\
F & 199 & 5,16 & 5,09 & 0,50 \\
G & 207 & 8,01 & 5,52 & 0,42 \\
H & 198 & 4,15 & 2,71 & 0,20 \\
\hline
\end{tabular}

Tab. II. Tamanho (ha) das áreas de vida de 14 machos de Cistothorus platensis (Latham, 1790) em campo sujo no Parque Nacional da Chapada dos Veadeiros, Goiás, Brasil central, em três períodos de 2008: período não-reprodutivo - estação chuvosa (PNREC), período não-reprodutivo - estação seca (PNRES) e período reprodutivo (PR). Os estimadores utilizados foram: Mínimo Polígono Convexo (MPC), Kernel 95\% e Kernel 50\%. O hífen (-) indica que as áreas não atingiram a estabilização ou que os indivíduos não foram avistados.

\begin{tabular}{|c|c|c|c|c|c|c|c|c|c|}
\hline \multirow{3}{*}{ Machos } & \multicolumn{9}{|c|}{ Métodos e Períodos } \\
\hline & \multicolumn{3}{|c|}{ Mínimo Polígono Convexo } & \multicolumn{3}{|c|}{ Kernel 95\% } & \multicolumn{3}{|c|}{ Kernel $50 \%$} \\
\hline & PNREC & PNRES & PR & PNREC & PNRES & PR & PNREC & PNRES & PR \\
\hline $\mathrm{A}$ & 1,29 & 4,38 & - & 1,29 & 3,87 & - & 0,21 & 0,61 & - \\
\hline $\mathrm{B}$ & 1,39 & 1,90 & 3,71 & 1,77 & 2,66 & 3,34 & 0,24 & 0,59 & 0,26 \\
\hline $\mathrm{C}$ & 1,09 & - & - & 1,64 & - & - & 0,24 & - & - \\
\hline $\mathrm{D}$ & 1,26 & - & - & 1,25 & - & - & 0,31 & - & - \\
\hline E & 1,27 & 3,77 & 1,25 & 1,65 & 2,85 & 1,55 & 0,32 & 0,32 & 0,27 \\
\hline $\mathrm{F}$ & 1,52 & 4,58 & 1,63 & 1,67 & 6,87 & 2,34 & 0,20 & 1,70 & 0,61 \\
\hline G & 2,04 & 6,16 & 4,25 & 2,37 & 6,61 & 5,31 & 0,16 & 0,48 & 0,51 \\
\hline $\mathrm{H}$ & 2,07 & 1,96 & 1,42 & 1,39 & 2,98 & 2,59 & 0,12 & 0,65 & 0,59 \\
\hline I & - & - & - & - & - & - & - & - & - \\
\hline $\mathrm{J}$ & - & 4,36 & 0,54 & - & 3,92 & 1,68 & - & 0,36 & 0,29 \\
\hline $\mathrm{K}$ & - & 4,02 & 1,40 & - & 5,12 & 1,74 & - & 0,63 & 0,42 \\
\hline $\mathrm{L}$ & - & 1,82 & - & - & 2,07 & - & - & 0,35 & - \\
\hline $\mathrm{M}$ & - & 0,92 & 0,24 & - & 1,92 & 1,44 & - & 0,32 & 0,22 \\
\hline $\mathrm{N}$ & - & 1,75 & 1,59 & - & 2,28 & 1,93 & - & 0,28 & 0,25 \\
\hline
\end{tabular}




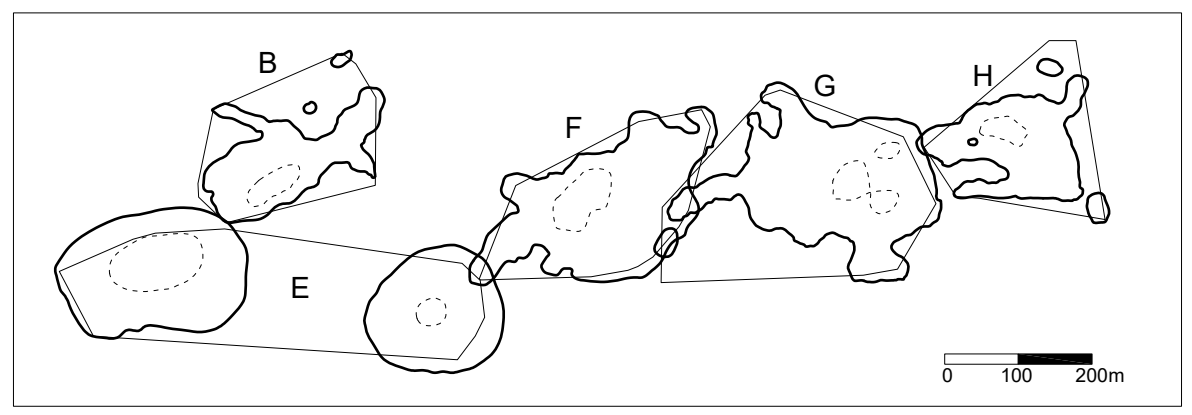

Fig. 3. Áreas de vida de cinco indivíduos de Cistothorus platensis (Latham, 1790) em trecho de campo sujo no Parque Nacional da Chapada dos Veadeiros, entre fevereiro e dezembro de 2008. As áreas de vida foram delimitadas pelo método do Mínimo Polígono Convexo (linha simples) e Kernel 95\% (linha espessa). As áreas nucleares foram representadas pelas linhas tracejadas.

Tab. III. Proporção (\%) da área de sobreposição entre as áreas de vida de 14 machos de Cistothorus platensis (Latham, 1790) em campo sujo no Parque Nacional da Chapada dos Veadeiros, Goiás, Brasil central, em três períodos de 2008: período não-reprodutivo - estação chuvosa (PNREC), período não-reprodutivo - estação seca (PNRES) e período reprodutivo (PR). As áreas de vida foram calculadas utilizando-se três estimadores: Mínimo Polígono Convexo (MPC), Kernel 95\% e Kernel 50\%. O hífen (-) indica que os indivíduos não foram avistados num certo período.

\begin{tabular}{|c|c|c|c|c|c|c|c|c|c|}
\hline \multirow{3}{*}{ Indivíduos } & \multicolumn{9}{|c|}{ Métodos e Períodos } \\
\hline & \multicolumn{3}{|c|}{ Mínimo Polígono Convexo } & \multicolumn{3}{|c|}{ Kernel 95\% } & \multicolumn{3}{|c|}{ Kernel $50 \%$} \\
\hline & PNREC & PNRES & PR & PNREC & PNRES & PR & PNREC & PNRES & PR \\
\hline A & 0,0 & 37,1 & - & 0,0 & 45,5 & - & 0,0 & 1,8 & - \\
\hline B & 0,0 & 57,5 & 3,6 & 0,0 & 59,8 & 22,9 & 0,0 & 4,1 & 0,0 \\
\hline $\mathrm{C}$ & 0,0 & - & - & 0,0 & - & - & 0,0 & - & - \\
\hline $\mathrm{D}$ & 1,2 & - & - & 2,1 & - & - & 0,0 & - & - \\
\hline E & 1,2 & 63,3 & 0,0 & 1,6 & 60,6 & 2,1 & 0,0 & 3,5 & 0,0 \\
\hline $\mathrm{F}$ & 0,0 & 0,6 & 0,0 & 0,0 & 10,3 & 0,0 & 0,0 & 0,0 & 0,0 \\
\hline G & 0,0 & 0,5 & 0,0 & 0,0 & 10,5 & 0,0 & 0,0 & 0,0 & 0,0 \\
\hline $\mathrm{H}$ & 0,0 & 23,6 & 0,0 & 0,0 & 30,5 & 0,0 & 0,0 & 0,0 & 0,0 \\
\hline I & 0,0 & - & - & 0,0 & - & - & 0,0 & - & - \\
\hline $\mathrm{J}$ & - & 66,9 & 89,7 & - & 79,0 & 64,7 & - & 6,6 & 0,0 \\
\hline K & - & 0,0 & 0,0 & - & 1,3 & 0,0 & - & 0,0 & 0,0 \\
\hline $\mathrm{L}$ & - & 25,4 & - & - & 41,3 & - & - & 0,0 & - \\
\hline $\mathrm{M}$ & - & 47,6 & 55,2 & - & 43,7 & 41,7 & - & 0,0 & 0,0 \\
\hline $\mathrm{N}$ & - & 26,6 & 30,5 & - & 22,6 & 66,1 & - & 0,0 & 0,0 \\
\hline
\end{tabular}

Para quatro $(\mathrm{B}, \mathrm{F}, \mathrm{G}, \mathrm{H})$ dos cinco indivíduos observados durante os três períodos de estudo, não foram verificadas mudanças nas suas posições na paisagem. Esses machos mantiveram suas áreas de vida na mesma localidade durante todo o período de estudo. Os demais indivíduos não foram vistos na mesma localidade em pelo menos um dos períodos de estudo. $\mathrm{O}$ macho $\mathrm{A}$ foi observado na mesma localização nas porções seca e chuvosa do período nãoreprodutivo, mas não foi encontrado no período reprodutivo. Os indivíduos $\mathrm{K}, \mathrm{M}$ e $\mathrm{N}$ não estavam na área de estudo no período não-reprodutivo - estação chuvosa. Nos períodos reprodutivo e não-reprodutivo - estação seca, passaram a ocupar a área de estudo e permaneceram nos mesmos locais durante as duas estações. Os machos C, D, I e L foram vistos em apenas um dos períodos estudados e estiveram ausentes da área de estudo nos demais períodos. Os indivíduos E e J mudaram as localizações de suas áreas de vida.

Houve substituição de indivíduos em algumas áreas. As áreas ocupadas pelos machos D e E no período não- reprodutivo - estação chuvosa passaram a ser utilizadas pelo indivíduo K nos períodos seguintes. No período reprodutivo, o indivíduo E utilizou a área anteriormente ocupada pelo macho A. Além disso, no período não-reprodutivo - estação seca, alguns indivíduos (E e N) foram observados em uma região não colonizada no período não-reprodutivo - estação chuvosa. Essa região havia sido queimada na estação seca do ano anterior (setembro de 2007) e coincidiu com o limite das áreas de vida dos indivíduos A, B, D e E no período não-reprodutivo - estação chuvosa.

Comportamento. Em todas as observações, os indivíduos de C. platensis voaram baixo e a curtas distâncias, no máximo $10 \mathrm{~m}$. Frequentemente cantavam quando estavam expostos em hastes de capim e pequenos arbustos. Foram observados emitindo também um chiado (similar a Xeno-canto XC427218), principalmente quando estavam escondidos na vegetação herbácea.

Os machos foram observados cantando ou chiando em grande parte dos pontos em que foram avistados. Encontros 
físicos agonísticos não foram observados. No entanto, verificou-se em 11 ocasiões que, quando um macho cantava, um outro macho que ocupava uma área adjacente parecia responder imediatamente e ambos permaneciam cantando por vários minutos, de maneira alternada.

Em algumas áreas de vida, os machos estavam acompanhados de outros indivíduos, provavelmente fêmeas. Ambos se deslocavam juntos, um atrás do outro, mantendo no máximo uma distância de $15 \mathrm{~m}$ entre eles. No período não-reprodutivo - estação chuvosa, sete dos oito machos que foram observados estavam pareados. No período nãoreprodutivo - estação seca, seis dos 11 machos estudados estavam pareados e, no período reprodutivo, todos os indivíduos permaneceram pareados.

O macho J foi observado somente a partir do período não-reprodutivo - estação seca. Entretanto, ele não deixava a cauda erguida, como é característico dos indivíduos desta espécie. Além disso, cantava de forma diferente, errando algumas notas típicas dos cantos de outros machos. $\mathrm{Na}$ estação reprodutiva, este indivíduo foi observado com a cauda erguida e cantando como os demais co-específicos. Tais comportamentos sugerem tratar-se de um jovem.

\section{DISCUSSĀO}

Área de vida e território. Neste estudo, os machos foram observados vocalizando em praticamente todos os pontos em que foram avistados e, grande parte das áreas de vida analisadas foram de uso exclusivo do macho e da suposta fêmea que o acompanhava.

Tamanho da área de vida. $O$ tamanho médio da área de vida de C. platensis foi maior que na região temperada. $\mathrm{O}$ valor médio foi de 0,1780 ha em Minnesota (BuRNs, 1982) e variou entre 0,042 ha e 0,2437 ha em Minnesota e Wisconsin (KIRSCH et al., 2007). As áreas de vida de C. platensis na região temperada foram estimadas somente na estação reprodutiva da espécie. Desta forma, cabe compará-las ao período equivalente na Região Neotropical. O tamanho da área de vida desta espécie na estação reprodutiva na Chapada dos Veadeiros foi, pelo menos, 10 vezes maior que as áreas de vida estimadas na América do Norte. Variações regionais na abundância de recursos alimentares e de co-específicos podem ser fatores contribuindo para tais diferenças entre campos naturais de regiões tropicais e temperadas.

Variação sazonal. A área de vida anual de C. platensis é muito maior que a sua área de vida no período reprodutivo. Ao longo das diferentes estações, foi observado que os machos tendem a parar de defender algumas porções do seu território para defender novas áreas. Tal fato ressalta a importância da realização de estudos em períodos além da estação reprodutiva para evitar subestimativas do tamanho das áreas de vida.

Foi verificada diferença significativa entre o tamanho das áreas de vida nos diferentes períodos estudados. As áreas de vida de C. platensis foram maiores na porção seca do período não-reprodutivo do que na estação chuvosa, que inclui uma parte do período não-reprodutivo e todo o período reprodutivo da espécie. Tal fato provavelmente está relacionado à maior produtividade de ambientes de cerrado sensu lato na estação chuvosa (GotTSBERGER \& SilberBaUERGotTsberger, 1983; Filgueiras, 2002; MACEDO, 2002). A abundância de insetos pertencentes a ordens como Isoptera, Coleoptera e Hemiptera tem seu pico durante a estação chuvosa (PINHEIRo et al., 2002), aumentando a base de presas das aves. A menor abundância de alimento em campos sujos na estação seca provavelmente contribui para um aumento de áreas de forrageamento, levando a áreas de vida maiores.

Houve baixa sobreposição entre as áreas de vida de indivíduos vizinhos. As áreas de vida foram ativamente defendidas através de intimidação por vocalização. BROWN (1964) propôs que um animal apenas estabelece um território quando os benefícios proporcionados por este são maiores do que os custos. Entre as vantagens de possuir um território, pode-se destacar a maior facilidade de acesso ao alimento, o aumento na chance de conseguir um parceiro para reprodução, a obtenção de um sítio apropriado para nidificação e a proteção da prole (BROWN, 1964; ADAMs, 2001). Entre os custos estão o risco de sofrer algum ferimento nas disputas territoriais e o gasto de tempo e energia na defesa do território (Brown, 1964; AdAMs, 2001). Estes fatores podem estar influenciando a distribuição de territórios de C. platensis em campos no Cerrado.

As sobreposições foram observadas principalmente na porção seca da estação não-reprodutiva, período de menor produtividade do Cerrado (GotTSBERGER \& SilberbaUERGottsberger, 1983; Filgueiras, 2002; Macedo, 2002; Pinheiro et al., 2002). Neste período, os alimentos devem estar concentrados em determinados locais, ou ocorrer em menor abundância, aumentando a competição por recursos limitados. O aumento da competição poderia aumentar o custo de defesa da área e reduzir os benefícios da manutenção do território, tendo como consequência uma maior sobreposição entre as áreas de vida. Em algumas ocasiões, foram observados indivíduos adentrando as áreas de vizinhos sem emitir qualquer vocalização. Desta forma, algumas das sobreposições podem também ter ocorrido devido à entrada fortuita de invasores em territórios estabelecidos.

O indivíduo que apresentou as maiores sobreposições com vizinhos era, provavelmente, um jovem. Este macho poderia ser um indivíduo flutuante (WINKER, 1998), tentando explorar áreas já ocupadas e inflando as sobreposições. Indivíduos flutuantes costumam apresentar áreas de vida móveis, com frequentes incursões em áreas ocupadas por co-específicos, alta sobreposição e amplas áreas de forrageamento (Brown \& SHERry, 2008). Além disso, geralmente são mais jovens do que os donos dos territórios (SERGio et al., 2009). Raramente um indivíduo flutuante tem sucesso em invadir e ocupar um território pré-estabelecido (HAHN \& BAUER, 2008) e, talvez por este motivo, tenha sido observada a mobilidade deste indivíduo entre estações, tentando se estabelecer em diferentes locais.

Indivíduos de C. platensis parecem evitar estabelecer seus territórios em áreas recentemente queimadas. Um trecho queimado dentro da área de estudo não foi ocupado por 
nenhum indivíduo até, pelo menos, sete meses depois da queimada. Esta resposta deve ocorrer porque esta espécie escolhe seus territórios, pelo menos em parte, baseada no grau de proteção que a vegetação oferece, apresentando preferência por locais com vegetação herbácea densa na América do Norte (BuRns, 1982). Em campos do sul do Brasil, esta espécie é mais encontrada em trechos com o estrato herbáceo mais alto que $35 \mathrm{~cm}$ (Dias et al., 2014). Assim, a queimada do estrato herbáceo no campo sujo estudado pode ter eliminado temporariamente componentes da vegetação que são importantes para C. platensis. Embora os efeitos das queimadas sobre os artrópodes possam ser positivos a longo prazo (ANDERSON et al., 1989; SiEMAN et al., 1997), logo após o fogo a abundância e a diversidade de artrópodes podem declinar temporariamente em savanas (SWENGEL, 2001). Com o desenvolvimento da estrutura herbácea alguns meses após o fogo, haveria aumento de artrópodes, tornando estas áreas mais adequadas a C. platensis.

Modo de vida. Diversos estudos apontam que as populações de $C$. platensis da América do Norte são migratórias e nômades (BURNS, 1982; LINGLE \& BEDELL, 1989; Del Hoyo et al., 2005; Kirsch et al., 2007) e que as populações das Américas Central e do Sul são residentes e sedentárias, apresentando alta fidelidade territorial (Kroodsma \& Verner, 1978; Kroodsma et al., 1999b, 2002; Del Hoyo et al., 2005). No presente trabalho, indivíduos de C. platensis foram avistados durante todo o período de estudo. Cinco machos permaneceram nas mesmas localidades durante todo o ano. No entanto, a alta substituição de indivíduos e as mudanças nas posições das áreas de vida de dois deles sugerem a existência de mobilidade. Dos 14 machos estudados, muitos $(n=9)$ não permaneceram na mesma localidade, o que pode representar dispersão, nomadismo ou até mesmo morte. Em um estudo realizado no México, GARZA \& ITA (2001) sugeriram a existência de nomadismo em uma população não-migratória de C. platensis. KroodsMA et al. (1999b) observaram que, apesar do abandono temporário de alguns territórios em áreas na Costa Rica, pelo menos um terço das aves anilhadas retornaram aos mesmos territórios na estação reprodutiva seguinte. Estudos mais longos seriam necessários para analisar se machos de C. platensis também retornam aos seus territórios no Brasil central.

Os indivíduos da população estudada parecem ser monogâmicos, assim como os das outras populações estudadas na América Central e do Sul (Kroodsma \& Verner, 1978; Kroodsma et al., 1999b, 2002; Del Hoyo et al., 2005). De fato, uma baixa taxa social de poliginia foi recentemente registrada em campos temperados da Argentina (Llambías et al., 2018). Na maioria das vezes, os machos e as fêmeas se moveram juntos e não foram observados mais de dois indivíduos por área de vida. Não houve nenhuma evidência da existência de uma relação social poligínica como comumente observada na América do Norte (CrAwFORD, 1977; BURNs, 1982).

Este foi o primeiro estudo sobre área de vida de $C$. platensis na Região Neotropical. Características observadas neste estudo como maiores áreas de vida e monogamia salientam a existência de diferenças substanciais entre as populações norte-americanas e tropicais. Estudos sobre a área de vida de C. platensis em outros locais na Região Neotropical são necessários para verificar se os padrões observados no Parque Nacional da Chapada dos Veadeiros se repetem.

Agradecimentos. Agradecemos à Universidade de São Paulo e à Universidade Federal Rural do Semi-Árido pelo suporte ao trabalho. Ao Cemave pela permissão para anilhamento. Ao Instituto Chico Mendes por permitir o estudo das aves no Parque Nacional da Chapada dos Veadeiros e aos funcionários J. F. S. Rebello, D. R. M. Borges e S. N. Fonseca por todo o apoio oferecido no parque. Também somos gratos a G. Freitas e A. Gabas pela ajuda na utilização do Arcview, e a M. Luz pela ajuda nas análises estatísticas. Esta pesquisa recebeu o apoio financeiro da FAPESP (05/00773-3), CNPq (471360/2006-6; 130315/2009-5) e Idea Wild. José C. Motta-Junior, L. E. Lopes, M. P. Castro Neves, M. A. Marini, R. A. Dias e um revisor anônimo contribuíram com numerosas sugestões a uma versão anterior deste manuscrito.

\section{REFERÊNCIAS}

Adams, E. S. 2001. Approaches to the study of territory size and shape. Annual Review of Ecology and Systematics 32(1):277-303.

Amaral, M. F. \& Macedo, R. H. F. 2003. Breeding patterns and habitat use in the endemic Curl-crested Jay of central Brazil. Journal of Field Ornithology 74(2):331-340.

ANDERSON, R. C.; LEAHY, T. \& DHILlion, S. 1989. Numbers and biomass of selected insect groups on burned and unburned sand prairie. American Midland Naturalist 122(1):151-162.

Assad, E. D. 1994. Chuva nos Cerrados. Análises e espacialização. Brasília, EMBRAPA Cerrados. 77p.

Brown, D. R. \& SHERry, T. W. 2008. Alternative strategies of space use and response to resource change in a wintering migrant songbird. Behavioural Ecology 19(6):1314-1325.

BRown, J. L. 1964. The evolution of diversity in avian territorial systems. Wilson Bullettin 76(1):160-169.

Brown, J. L. \& OrIans, G. H. 1970. Spacing patterns in mobile animals. Annual Review of Ecology and Systematics 1:239-262

BurNs, J. T. 1982. Nests, territories, and reproduction of Sedge Wrens (Cistothorus platensis). Wilson Bulletin 94(2):338-349.

BuRT, W. H. 1943. Territoriality and home range concepts as applied to mammals. Journal of Mammalogy 24(2):346-352.

CARPenter, F. L. 1987. The study of territoriality: complexities and future directions. American Zoologist 27(2):401-409.

Castro, E. A. \& Kauffman, J. B. 1998. Ecosystem structure in the Brazilian Cerrado: a vegetation gradation of aboveground biomass, root biomass and consumption by fire. Journal of Tropical Ecology 14(1):263-283.

CraWford, R. D. 1977. Polyginous breeding of Short-Billed Marsh Wrens. Auk 94(2):359-362.

Del Hoyo, J.; Elliott, A. \& Christie, D. 2005. Handbook of the birds of the world. Cuckoo-Shrikes to Thrushes. Barcelona, Lynx Edicions, v. $10.895 \mathrm{p}$.

De Solla, S.; Bonduriansky, R. R. \& BRooks, R. J. 1999. Eliminating autocorrelation reduces biological relevance of home range estimates. Journal of Animal Ecology 68(1):221-234.

Dias, R. A.; Bastazini, V. A. G. \& Gianuca, A. T. 2014. Bird-habitat associations in coastal rangelands of southern Brazil. Iheringia, Série Zoologia 104(2):200-208.

DuCA, C. \& MarinI, M. A. 2014. Territorial system and adult dispersal in a cooperative-breeding tanager. The Auk 131(1):32-40.

Eiten, G. 1972. The cerrado vegetation of Brazil. Botanical Review 38(1):205-341.

ESRI. 1999. Getting to know Arc View GIS: the geographic information system (GIS) for everyone. Redlands, Prentice-Hall.

FelfiLI, J. M.; SilvA, M. C. \& Rezende, A. V. 2007. Biogeografia do bioma cerrado: vegetação e solos da Chapada dos Veadeiros. Brasília, Editora Universidade de Brasília. 324p. 
Filgueiras, T. S. 2002. Herbaceous plant communities. In: Oliveira, P. S. \& MARQUIS, R. J. eds. The Cerrados of Brazil: ecology and natural history of a Neotropical Savanna. New York, Columbia University Press, p. 121-139.

Freitas, G. H. S. \& Rodrigues, M. 2012. Territory distribution and habitat selection of the Serra Finch (Embernagra longicauda) in Serra do Cipó, Brazil. The Wilson Journal of Ornithology 124(1):57-65.

Garza, H. G. S. \& Ita, A. O. 2001. Posible nomadismo em el Chivirín sabanero (Cistothorus platensis) em el Distrito Federal, México. Huitzil 2(1):15.

Gottsberger, G. \& Silberbauer-Gottsberger, I. 1983. Dispersal and distribution in the Cerrado vegetation of Brazil. Sonderbänd des Naturwissenschaftlichen Vereins in Hamburg 7(2):315-352.

Hahn, S. \& Bauer, S. 2008. Dominance in feeding territories relates to foraging success and offspring growth in brown skuas Catharacta antartica lonnbergi. Behavioural Ecology and Sociobiology 62(6): 1149-1157.

Harris, S.; Cresswell, W. J.; Forde, P. G.; Trewhella, W. J.; Woollard, T. \& WraY, S. 1990. Home-range analysis using radiotracking data - a review of problems and techniques particularly as applied to the study of mammals. Mammalian Review 20(1):97-123.

Hooge, P. N. \& Eichenlaub, B. 2000. Animal movement extension for Arcview. Anchorage, USGS, Alaska Science Center, v. 2.04.

ICMBio. 2019. Parque Nacional da Chapada dos Veadeiros. Disponível em: <http://www.icmbio.gov.br/parna_veadeiros $>$. Acessado em: 26 de janeiro de 2019.

JACOB, A. A. \& RUDRAN, R. 2003. Radiotelemetria em estudos populacionais. In: Cullen, L.; Rudran, R. \& Valladares-Pádua, C. eds. Métodos de estudo em biologia da conservação e manejo da vida silvestre. Curitiba, Editora UFPR, p. 285-342.

Kirsch, E. M.; Gray, B. R.; Fox, T. J. \& Thogmartin, W. E. 2007. Breeding bird territory placement in riparian wet meadows in relation to invasive Reed Canary Grass, Phalaris arundinaceae. Wetlands 27(3):644-655.

Kroodsma, D. E.; Liu, W. C.; Goodwin, E. \& Bedell, P. E. 1999a. The ecology of song improvisation as illustrated by North American Sedge Wrens. Auk 116(2):373-386.

Kroodsma, D. E.; SAnchez, J.; Stemple, D. W.; Goodwin, E.; Da Silva, M. L. \& Vielliard, J. M. E. 1999b. Sedentary life style of neotropical Sedge Wrens promotes song imitation. Animal Behaviour 57(4):855-863.

Kroodsma, D. E. \& Verner, E. J. 1978. Complex singing behaviors among Cistothorus wrens. Auk 95(4):703-716.

Kroodsma, D. E.; Woods, R. W. \& Goodwin, E. A. 2002. Falkland Island Sedge Wrens (Cistothorus platensis) imitate rather than improvise large song repertories. Auk 119(3):523-528.

Laver, P. N. \& Kelly, M. J. 2008. A critical review of home range studies. Journal of Wildlife Management 7(2):290-298.

Llambías, P. E.; Garrido, P. S.; Jefferies, M. M. \& Fernández, G. J. 2018. Social mating system, male parental care contribution and life history traits of a southern Sedge Wren (Cistothorus platensis platensis) population: a comparison with northern Sedge Wrens (Cistothorus platensis stellaris). Journal of Ornithology 159(1):221-231.

Lingle, G. R. \& Bedell, P. 1989. Nesting ecology of Sedge Wrens in Hall County, Nebraska. Nebraska Bird Review 57(1):47-49.

Lopes, L. E. \& MARINI, M. A. 2006. Home range and habitat use by Suiriri affinis and Suiriri islerorum (Aves: Tyrannidae) in the central Brazilian Cerrado. Studies on Neotropical Fauna and Environment 41(2):8792.

MACEDO, R. H. F. 2002. The avifauna: ecology, biogeography, and behavior. In: Oliveira, P. S \& MArquis, R. J. eds. The Cerrados of Brazil: ecology and natural history of a Neotropical Savanna. New York, Columbia University Press, p. 242-265.

Marini, M. A.; Borges, F. J. A.; Lopes, L. E.; Sousa, N. O. M.; Gressler, D. T.; Santos, L. R.; Paiva, L. V.; DuCA, C. G.; Manica, L.T.; Rodrigues,
S. S.; França, L. F.; Costa, P. M.; França, L. C.; Heming, N. M.; Silveira, M. B.; Pereira, Z. P.; Lobo, Y. P. P.; Medeiros, R. C. S. \& ROPER, J. J. 2012. Breeding biology of birds in the cerrado of Central Brazil. Ornitología Neotropical 23(3):385-405.

Odum, E. P. \& Kuenzler, E. J. 1955. Measurement of territory size and home range size in birds. Auk 72(1):128-137.

Oliveira, P. E. 1998. Fenologia e biologia reprodutiva das espécies de Cerrado. In: Sano, S. M. \& Almeida, S. P. eds. Cerrado, ambiente e flora. Planaltina, Embrapa, p. 169-194.

Oliveira-Filho, A. T. \& RatTer, J. A. 2002. Vegetation physiognomies and woody flora of the Cerrado biome. In: OliveIra, P. S. \& MARQuis, R. J. eds. The Cerrados of Brazil: ecology and natural history of a Neotropical Savanna. New York, Columbia University Press, p. 91-120.

Pinheiro, F.; Diniz, I. R. \& Bandeira, M. P. S. 2002. Seasonal pattern of insect abundance in the Brazilian cerrado. Austral Ecology 27(2):132136.

Preston, K. L.; Mock, P. J.; Grishaver, M. A.; Bailey E. A. \& King, D. F. 1998. California Gnatcatcher territorial behavior. Western Birds 29(2):242-257.

Powell, R. A. 2000. Animal home ranges and territories and home ranges estimators. In: BoITANI, L. \& FULLER. T. eds. Research techniques in animal ecology: controversies and consequences. New York, Columbia University Press, p. 65-110.

Pyke, G. H.; Pulliam, H. R. \& Charnov, E. L. 1977. Optimal foraging: a selective review of theory and tests. Quarterly Review of Biology 52(1):137-154.

Ribeiro, J. F. \& Walter, B. M. T. 1998. Fitofisionomias do bioma Cerrado. In: Sano, S. M. \& Almeida, S. P. eds. Cerrado: ambiente e flora. Planaltina, EMBRAPA Cerrados, p. 89-166.

Seaman, D. E.; Millspaugh, J. J.; Kernohan, B. J.; Brundige, G. C.; RAEDEKe, K. J. \& Gitzen, R. A. 1999. Effects of sample size on Kernel home range estimates. Journal of Wildlife Management 63(3):739-747.

Sergio, F.; Blas, J. \& Hiraldo, F. 2009. Predictor of floater status in a long-lived bird: a cross sectional and longitudinal test hyphoteses. Animal Ecology 78(1):109-118.

Sick, H. 1997. Ornitologia Brasileira. Rio de Janeiro, Nova Fronteira. 862p.

Siemann, E.; HaArstad, J. \& Tilman, D. 1997. Short-term and long-term effects of burning on oak savanna arthropods. American Midland Naturalist 137(2):349-361.

Silva, S. R.; Silva, A. P.; Munhoz, C. B.; Silva JR, M. C. \& Medeiros, M. B. 2001. Guia de plantas do Cerrado utilizadas na Chapada dos Veadeiros. Brasília, WWF-Brazil. 123p.

Souza, D. C.; Vieira, L. D. \& Castro, A. L. S. 2018. Territoriality and home range of the Red-legged Seriema (Cariama cristata). Ornitologia Neotropical 29(1):101-105.

Swengel, A. B. 2001. A literature review of insect responses to fire, compared to other conservation managements of open habitat. Biodiversity \& Conservation 10(6):1141-1169.

Tubelis, D. P. \& CaVALCANTI, R. B. 2001. A comparison of bird communites in natural and disturbed non-wetland open habitats in the Cerrado's central region, Brazil. Bird Conservation International 10(2):331-350.

VANDER WERF, E. A. 1998. Breeding biology and territoriality of the Hawaii Creeper. Condor 100(2):541-545.

Vickery, P. D.; Tubaro, P. L.; Silva, J. M. C.; Peterjohn, B. G.; Herkert, J. R. \& CaVAlCanti, R. B. 1999. Conservation of grassland birds in the Western Hemisphere. Studies in Avian Biology 19(1):2-26.

WINKER, K. 1998. The concept of floater. Ornitologia Neotropical 9(2):111119.

Worton, B. J. 1987. A review of models of home range for animal movement. Ecological Modelling 38(2):277-298.

ZAR, J. L. 2009. Biostatistical analysis. Harlow, Pearson. 960p. 